\title{
Method to Increase the Systemically Delivered Amount of Drug from Dissolving Microneedles
}

\author{
Yukako Ito,* Noriyuki Hamasaki, Hirofumi Higashino, Yuki Murakami, Nao Miyamoto, and \\ Kanji Takada*
}

Department of Pharmacokinetics, Kyoto Pharmaceutical University; Yamashina-ku, Kyoto 607-8412, Japan. Received May 22, 2012; accepted September 8, 2012

To increase the absorbed amount of a drug from dissolving microneedles (DMs), three DM array chips were prepared in which (1) the drug was localized at the acral portion of DMs, (2) the drug was loaded in each whole DM, and (3) the drug was loaded in DMs and the chip. Fluorescein free form (FL) and its sodium salt (FLNa) were used as model drugs. The DM array chip had $15-\mathrm{mm}$ diameter with 225 DMs, each $500-\mu \mathrm{m}$ long with a $300-\mu \mathrm{m}$ diameter base. The respective FLNa contents in the three chips were (1) $0.18 \pm 0.03,(2)$ $0.64 \pm 0.07$, and (3) $10.95 \pm 1.07 \mathrm{mg}$. The FL contents were (1) $0.20 \pm 0.01$, (2) $0.68 \pm 0.03$ and (3) $12.47 \pm 1.01 \mathrm{mg}$. The in vitro release of fluorescein from FLNa DMs was faster than that from FL DMs. In vitro permeability experiments showed that (3) produced the greatest increase in the permeability of fluorescein through rat skin, especially in FLNa loaded DMs. In vivo rat absorption study by application of DM array chips to the rat abdominal skin for $6 \mathrm{~h}$ demonstrated that the systemically absorbed amount of fluorescein increased from $0.18 \pm 0.02 \mathrm{mg}, 0.53 \pm 0.19 \mathrm{mg}$, to $5.38 \pm 1.99 \mathrm{mg}$ from systems (1) and (2)-(3). By decreasing the application time of DMs to the rat skin, the absorbed amount of fluorescein decreased along with the application time. The physiological state of the skin recovered within $30 \mathrm{~min}$ after chip removal. Using a type (3) DM array chip, more than $1.0 \mathrm{mg}$ of water-soluble drug can be delivered to the systemic circulation.

Key words systemic delivery; dissolving microneedle; percutaneous absorption; rat

Transdermal drug delivery systems (TDDS) are attractive drug delivery systems (DDS) for the systemic delivery of drugs with high safety. ${ }^{1)}$ However, TDDS products of drugs are only nine compounds such as nicotine, fentanyl, and hormones. The main reason is the poor permeability of drugs through the human skin. Moreover, most drugs do not permeate through the skin at therapeutically relevant rates. Consequently, a therapeutic drug concentration is not maintained in systemic circulation. To increase the permeability of the drug through the skin, several methods such as chemical enhancers, electric fields, ultrasound, and thermal methods have been attempted. ${ }^{2-6)}$ Nevertheless, those technologies have not been applied to drugs as TDDS, because they often show skinrelated side-effects, skin damage, etc. To overcome the limitations of those technologies, microneedles (MNs) are being studied $^{7)}$ : small needles by which microconduits are formed on the skin surface and through which drugs are absorbed into the skin. Human skin comprises three layers. The outermost one is the stratum corneum that is dead tissue with thickness of $10-15 \mu \mathrm{m}$. The stratum corneum has a strong primary barrier function against exogenous compounds, including drugs. MNs physically destroy the stratum corneum. Consequently, the drug permeates through the skin. MNs do not cause pain. Now, MNs of four kinds are used ${ }^{8-10)}$ : (1) extremely small needles through which the drug solution can be injected into the skin; (2) metallic and/or silastic MNs onto which surface drug is coated; (3) metallic and/or silastic MNs by which microconduits are made on the skin, after which a drug solution or cream is applied following removal of the microneedle; and (4) dissolving microneedles (DMs). ${ }^{11)}$ DMs are made of watersoluble biopolymers such as chondroitin sulfate, hyaluronic acid and dextran, in which drug molecules are formulated as a solid dispersion or suspension. ${ }^{12)}$ An important advantage of

The authors declare no conflict of interest.
DMs over other MNs is that DMs have high biocompatibility, because the base polymer is made of water-soluble biopolymers and is more compatible with the human body than metal and silastic MNs.

We have been studying on DMs for use as a delivery system for hydrophilic drugs such as peptide protein drugs. Results of those studies have improved the bioavailability (BA) of macromolecular drugs having poor permeability through the skin, for example $91.3-97.7 \%$ for insulin in mice ${ }^{13)}$ and of $81.5-102.3 \%$ for low molecular weight heparin (LMWH) in rats, ${ }^{14)} 87.5 \%$ for recombinant human growth hormone (rhGH) in rats $^{15}$ ) and of $82.1-99.4 \%$ for erythropoietin (EPO) in mice. $^{16)}$ The relative BA of interferon (IFN) against subcutaneous injection of IFN solution was $79.9-117.8 \%$ in rats. ${ }^{17)}$ The relative physiological availability (PA) of insulin was 90-99\% in dogs. ${ }^{18)}$ The secondary stage of our DM study used a twolayered DM array chip of $1.0 \times 1.0 \mathrm{~cm}$, on which $100 \mathrm{DMs}$ with 10 lines and 10 columns were designed and prepared. ${ }^{19)}$ Each DM had $500 \mu \mathrm{m}$ length and $300 \mu \mathrm{m}$ diameter at its base. The drug was formulated at the acral portion of the DMs, which was inserted into the region of the epidermis and/or epidermal/dermal junctions by pressing with fingers. Two-layered DMs were applied to not only biopharmaceuticals but also hydrophilic low molecular weight (MW) drugs, desmopressin, ${ }^{20)}$ leuprolide, ${ }^{21)}$ and sumatriptan ${ }^{22)}$ where high BAs, 84.2-95.1\%, were obtained. However, the formulated amount of drug in DMs was limited to be $1.0 \mathrm{mg}$ at most. Most clinically useful drugs have the clinical dosages greater than $1.0 \mathrm{mg}$. Therefore, the need exists to formulate much drug in DMs. To achieve this purpose, we designed a DM array chip derivative that contains the drug not only in DM itself but also in the chip and the effect of the application time of DMs to the skin on the delivered amount of drug into the systemic circulation has been studied in both in vitro rat skin permeability experiment and in vivo rat absorption experiments. 


\section{Experimental}

Materials Sodium fluorescein (uranine, FLNa), fluorescein free form (FL), cellulose acetate (CA), and hydroxypropyl cellulose (HPC) were obtained from Wako Pure Chemical Industries, Ltd. (Osaka, Japan). Sodium chondroitin sulfate and evans blue (EB) were obtained from Nacalai Tesque Inc. (Kyoto, Japan). Lactate dehydrogenase (LDH) assay kit, cytotoxicity detection kit ${ }^{\text {PLUS }}$, was obtained from Roche Diagnostics GmbH (Manheim, Germany). Male Wistar Hannover rats used in the study were obtained from Japan SLC Inc. (Hamamatsu, Japan). A standard solid-meal commercial food (LabDiet, Nousan Corp., Yokohama, Japan) was used. All other materials used were of reagent grade and were used as received.

Preparation of DM Array Chips. Two-Layer DM Array Chip To $5 \mathrm{mg}$ of FLNa or FL as model drug and $10 \mathrm{mg}$ of sodium chondroitin sulfate, $30 \mu \mathrm{L}$ of distilled water was added. After kneading at room temperature, the mixture was degassed under reduced pressure and dispensed into a mold containing 225 inverted cone-shaped wells with an area of approximately $1.0 \mathrm{~cm}^{2}$. Each well had depth of $500 \mu \mathrm{m}$ and $300 \mu \mathrm{m}$ diameter at its top. The drug glue was filled into the wells. The mold was covered with a $300 \mathrm{~g}$ steel plate and dried well. After the plate was removed, glue made of $15 \mathrm{mg}$ of sodium chondroitin sulfate and $25 \mathrm{~mL}$ of distilled water was filled into the well and was also dried under pressure for $3 \mathrm{~h}$ using a steel plate. Then, glue was painted over a $1.7-\mathrm{cm}-$ diameter chip that was $2.5 \mathrm{~mm}$ thick. It was prepared using a tabletting machine (Handtab-100; Ichihashi Seki, Kyoto, Japan) with a mixture of CA and HPC $(10: 1)$. It was then dried for $3 \mathrm{~h}$ under the pressure of the stainless steel plate. Thereafter, the plate was removed and two-layered DM array chip was obtained by detachment with the chip.

One-Layer DM Array Chip As in the case of two-layered DM array chip, drug glue was also filled into the wells. The mold was covered with a $300 \mathrm{~g}$ steel plate and dried well. After the plate was removed, drug glue was once more filled into the well and was also dried under pressure by a steel plate for $3 \mathrm{~h}$. Then, glue was painted over a chip and the chip was attached to the mold. It was then dried under pressure of the stainless steel plate for $3 \mathrm{~h}$. Thereafter, the plate was removed and the one-layered DM array chip was obtained by detachment with the chip.

One-Layer DMs Formed on Drug Loaded Chip Five hundred grams of FLNa or FL was mixed well with $250 \mathrm{mg}$ of $\mathrm{CA}$ and of which mixture, $200 \mathrm{mg}$, was filled into the mortar of the tabletting machine. Next, $200 \mathrm{mg}$ of the mixture of CA and HPC (10:1) was also filled into the mortar and two-layered tablet was prepared. One-layer DM arrays were formed on thus obtained two-layered tablet.

Preparation of Drug Solution FLNa, $10 \mathrm{mg}$, was dissolved with $10 \mathrm{~mL}$ of phosphate buffered saline (PBS) and injection solution was obtained, $1.0 \mathrm{mg} / \mathrm{mL}$.

Drug Contents in DM Array Chips FLNa and FL extracted from DM array chips were dissolved with $1.0 \mathrm{~mL}$ of $0.1 \mathrm{~N}-\mathrm{NaOH}$ solution. Thereafter, $20 \mathrm{~mL}$ of PBS was also added. The extracted solution was diluted 20 times with PBS and fluorescein content was measured using a spectrofluorometer, Shimazdu RF-1500 (Shimadzau, Kyoto, Japan) where the excitation and emission wavelengths were 494 and $521 \mathrm{~nm}$.

In Vitro Dissolution Experiment of Fluorescein from
DM Array Chips In vitro dissolution experiments were performed using DM array chips with $20.0 \mathrm{~mL}$ of PBS, pH 7.4, as the dissolution medium at $37^{\circ} \mathrm{C}$. To determine the amount of fluorescein released from DM array chip, $0.2 \mathrm{~mL}$ of the dissolution medium was collected for the assay for $90 \mathrm{~min}$ at the predetermined time, $0,20,40 \mathrm{~s}$ and $1.0,1.5,2.0,2.5,3.0,4.0$, $5.0,10.0,15.0,30.0,45.0,60.0$, and $90.0 \mathrm{~min}$ and thereafter replaced with fresh dissolution medium that had been degassed earlier. In the case of FL loaded DM array chip, the following sampling times were added: 2, 4, 6 and $24 \mathrm{~h}$. The cumulative released amount of fluorescein from DM array chip was defined using the following equation.

$$
\text { cumulative amount released }=\left(\sum_{t=0}^{t} M_{t} / M_{\text {last }}\right) \times 100 \%
$$

Therein, $M_{t}$ is the amount of drug dissolved at each sampling time $t$, and $M_{\text {last }}$ is the dissolved amount of fluorescein at $90 \mathrm{~min}$ or $24 \mathrm{~h}$ after the start of the dissolution experiment.

In Vitro Skin Permeation Experiment with DMs Male Wistar rats, $311 \pm 23 \mathrm{~g}$, were anesthetized using an intraperitoneal (i.p.) injection of sodium pentobarbital: $50.0 \mathrm{mg} / \mathrm{kg}$. After the hair of the abdominal skin had been carefully removed, skin specimens (full-thickness, $3.0 \times 3.0 \mathrm{~cm}^{2}$ each) were freshly excised from the abdominal region of the rats. The subcutaneous tissue was trimmed and mounted on the Franz-type diffusion cell thermostated at $23^{\circ} \mathrm{C} .{ }^{23)} \mathrm{DMs}$ were applied to the epidermal surface of the skin. The receptor compartment was filled with $20.0 \mathrm{~mL}$ of PBS, $\mathrm{pH}$ 7.4. Permeation experiments were performed at $37^{\circ} \mathrm{C}$ and stirred at $100 \mathrm{rpm}$ throughout the experiment. After the start of the experiment, sample of $0.35 \mathrm{~mL}$, were withdrawn from the receptor compartment over $6 \mathrm{~h}$ and were replaced immediately with an equal volume of fresh buffer equilibrated at $37^{\circ} \mathrm{C}$. The obtained samples were centrifuged for $2 \mathrm{~min}$ at $14000 \mathrm{rpm}$ and the supernate was used immediately for fluorescein assay.

In Vivo Absorption Experiments in Rats Male Wistar Hannover rats, $331 \pm 17 \mathrm{~g}$, were anesthetized with an i.p. injection of sodium pentobarbital, $50.0 \mathrm{mg} / \mathrm{kg}$. One group consisted of 3-4 rats. At $5 \mathrm{~min}$ before drug administration, $0.25 \mathrm{~mL}$ of blank blood samples was obtained from the left jugular vein with a heparinized syringe. The hair on the abdominal region was carefully removed with a shaver. DMs were administered to the skin with an applicator described in our previous report $^{24)}$ and were pressed for $3.0 \mathrm{~min}$. At $0.5,1.0,1.5,2.0$, $2.5,3.0,4.0,5.0,6.0$, and $8.0 \mathrm{~h}$ after administration, $0.2 \mathrm{~mL}$ of blood samples was collected from the left jugular vein. By centrifuging at $12000 \mathrm{rpm}$ for $10 \mathrm{~min}$ at $4^{\circ} \mathrm{C}$ using a centrifuge (Kubota 1700, Kubota Corp., Tokyo, Japan), $100 \mu \mathrm{L}$ plasma samples were obtained. The resultant plasma samples were stored at $-80^{\circ} \mathrm{C}$ until analysis.

After a $0.25-\mathrm{mL}$ blank blood sample was obtained from the left jugular vein, the intravenous (i.v.) injection experiment was performed. Fluorescein solution was injected to the right jugular vein of rats, $1.0 \mathrm{mg} / \mathrm{kg}$, blood samples of $0.25 \mathrm{~mL}$ were obtained for $4 \mathrm{~h}$ using heparinized syringes. After centrifugation, plasma samples were obtained. All these plasma samples were frozen immediately in a deep freezer at $-80^{\circ} \mathrm{C}$ until analysis.

Safety Study of the DM Administered Rat Skin Before the administration of DM array chips to the rat skin, wet 
(1)

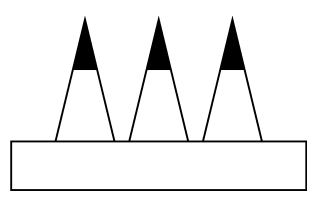

(2)

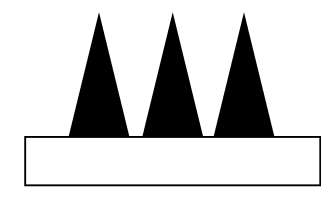

(3)

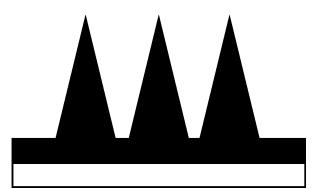

Fig. 1. Dissolving Microneedle (DM) Array Chips of Three Kinds Prepared in This Study

(1) Drug is localized at the acral portion of DMs; (2) drug is loaded in the whole DMs; and (3) drug is loaded both DMs and chip. The portions where drug is formulated are shown by black color.

tissue paper, $1.0 \times 1.0 \mathrm{~cm}$, was put on the skin for $1 \mathrm{~min}$. Thereafter, the paper was recovered and kept in a refrigerator till the assay of LDH. Placebo DMs which did not contain fluorescein dyes were administered to the rat skin and were kept on the skin for $3 \mathrm{~min}$ and $6 \mathrm{~h}$. Thereafter, tissue paper was put on the administered skin for $1 \mathrm{~min}$ and recovered at 15, 30, 45, 60, 90 and $120 \mathrm{~min}$. With thus obtained paper, LDH was extracted with $0.5 \mathrm{~mL}$ of PBS and LDH contents were measured. In another groups of rats, the skin pathology was studied by staining with $1.0 \%$ evans blue solution. The skin was recorded by a digital camera, Nikon D200 (Nikon, Tokyo, Japan).

All animal protocols were approved by the institutional animal care and use committee. Experiments were conducted in accordance with the Guidelines for Animal Experimentation, Kyoto Pharmaceutical University.

Assay Methods of Fluorescein in Plasma Samples To the obtained plasma sample, five times of methanol was added for deproteinization. After centrifugation at $12000 \mathrm{rpm}$ for $10 \mathrm{~min}$ at $4^{\circ} \mathrm{C}$, the supernate was obtained. By diluting the supernate with PBS, fluorescein content in the plasma was measured at the excitation and emission wavelengths of 494 and $521 \mathrm{~nm}$, respectively.

Pharmacokinetic Analysis Pharmacokinetic parameter values were calculated using a noncompartment analysis method. The maximum drug concentration, $C_{\max }$, and the time to reach maximum concentration, $T_{\max }$, were determined from the authentic plasma drug concentration $v s$. time data. The area under the plasma drug concentration $v s$. time curve, $A U C$, after percutaneous administration or i.v. injection was calculated using the linear trapezoidal rule up to the last measured drug concentration. The amount of drug absorbed into the systemic circulation was calculated by comparing the two $A U C \mathrm{~s}, A U C_{\text {chip }}$ and $A U C_{\text {i.v. }}$ that were obtained after

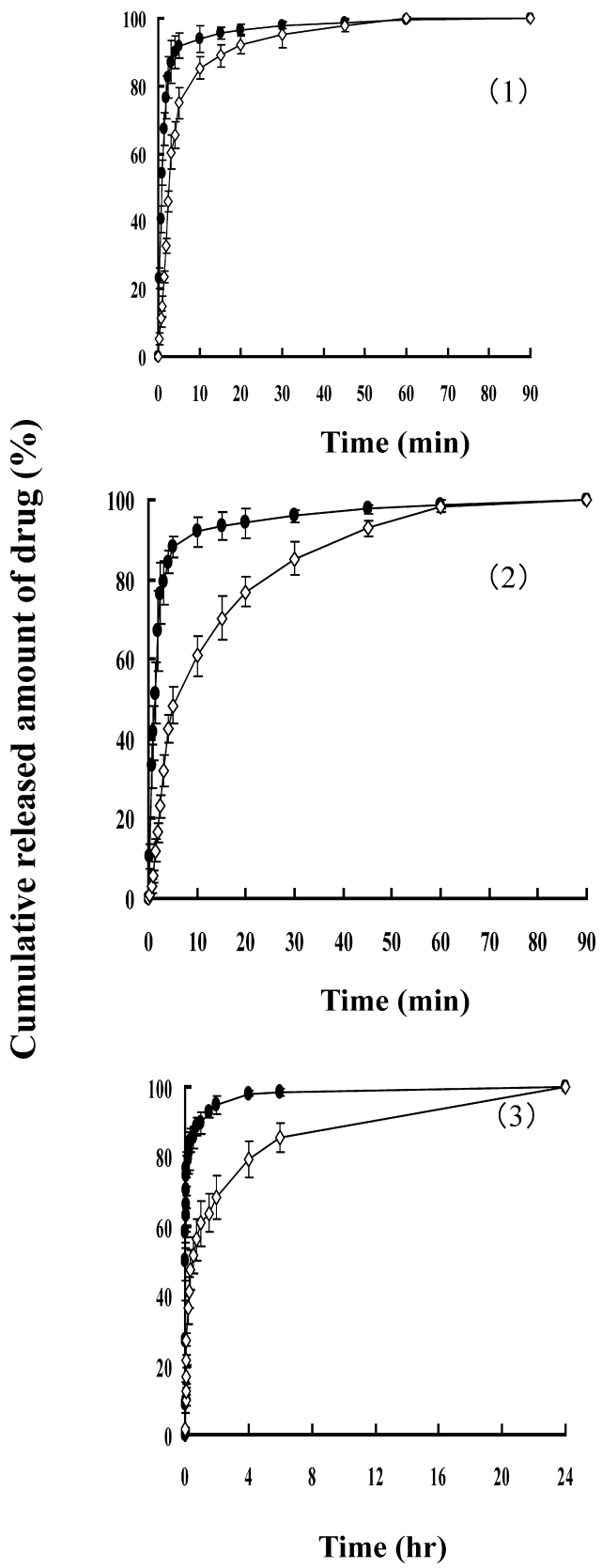

Fig. 2. Release Profile of Fluorescein, Model Drug, from DM Array Chips of Three Kinds

(1) Drug is localized at the acral portion of DMs; (2) drug is loaded in the whole DMs; and (3) drug is loaded both DMs and the chip. Sodium fluorescein loaded DM array chip (FLNa DM). $\diamond:$ Fluorescein loaded DM array chip (FL DM). Each point represents the mean \pm S.D. of 4 experiments.

percutaneous administration of FLNa loaded DM array chip and i.v. injection of fluorescein solution of which doses were Dose $_{\text {i.v. }}$ and Dose chip , respectively. The bioavailability (BA) of fluorescein from DM array chip was calculated using the following equation,

$$
\mathrm{BA}(\%)=\left(A U C_{\text {chip }} / A U C_{\text {i.v. }}\right) \times\left(\text { Dose }_{\text {i.v. }} / \text { Dose }_{\text {chip }}\right) \times 100
$$

Statistics All values are expressed as their mean \pm S.E. Statistically significant differences were assumed to be significant for $p<0.05$ (Student's unpaired $t$-test). 


\section{Results}

Drug Contents in DM Microneedle Array Chips Figure 1 shows that three kinds of FLNa or FL loaded DM array chips were prepared: (1) drug is localized at the acral portion of DMs, (2) drug is loaded in the whole DMs and (3) drug is loaded both DMs and chip. The FLNa contents in each DM array chip were, respectively, (1) $0.18 \pm 0.03 \mathrm{mg}$, (2) $0.64 \pm$ $0.07 \mathrm{mg}$ and (3) $10.95 \pm 1.07 \mathrm{mg}$. In addition, FL contents in each DM array chip were, respectively, (1) $0.2 \pm 0.01 \mathrm{mg}$, (2)

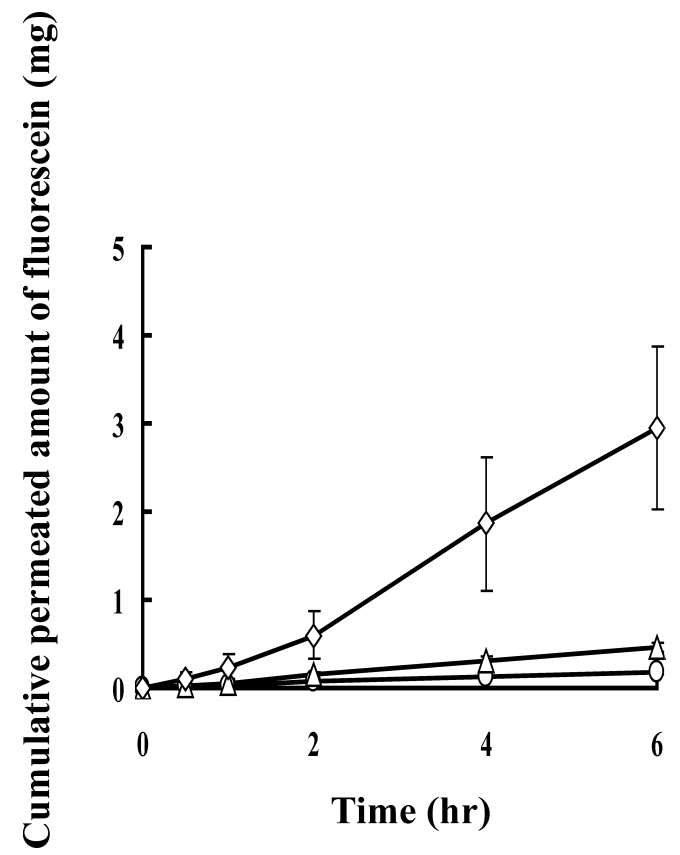

Fig. 3. In Vitro Permeation Profiles of Fluorescein through the Rat Skin from FLNa DMs

O: type (1) DMs, $\triangle$ : type (2) DMs, $\diamond$ : type (3) DMs. Each point represents the mean \pm S.D. of five experiments.

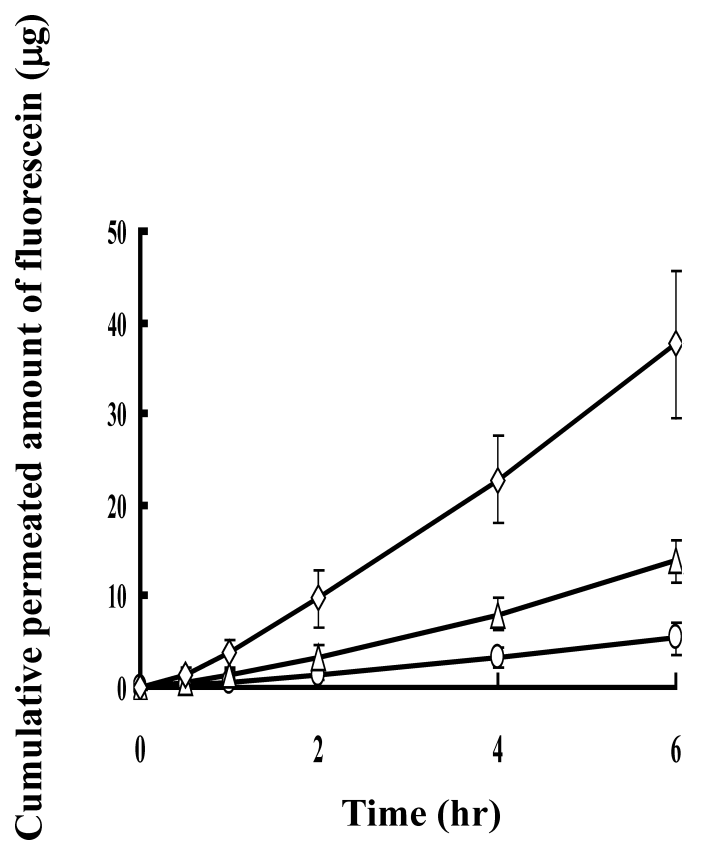

Fig. 4. In Vitro Permeation Profiles of Fluorescein through the Rat Skin from FL DMs

O: type (1) DMs, $\triangle$ : type (2) DMs, $\diamond$ : type (3) DMs. Each point represents the mean \pm S.D. of five experiments.
$0.68 \pm 0.03 \mathrm{mg}$, and (3) $12.47 \pm 1.01 \mathrm{mg}$.

Dissolution Rate of Fluorescein from DM Array Chips Figure 2 shows results of the dissolution experiment of FLNa and FL loaded three kinds of DM array chips. The dissolution rate of fluorescein from FLNa DMs was faster than that of FL DMs in the DM array chips of three kinds. Because the loaded space increased from the acral portion, type (1) DMs, to the base chip, type (3) DMs, the time when $100 \%$ of the loaded FLNa was released prolonged to $30 \mathrm{~min}, 45 \mathrm{~min}$ and 4h. On the other hand, the time when $100 \%$ of the loaded FL was released was $1 \mathrm{~h}$ for type (1) DMs, $1.5 \mathrm{~h}$ for type (2) DMs and $24 \mathrm{~h}$ for type (3) DMs, respectively. Consequently, the dissolution rate of fluorescein from FL loaded DMs was slower than FLNa loaded DM array chip.

In Vitro Permeability of Fluorescein through Rat Skin Three kinds of DMs containing FLNa and FL were administered to the isolated rat skin and in vitro permeability experiment was performed. Figures 3 and 4 show the results. The cumulative permeated amount of fluorescein through the rat

Table 1. Permeability of Fluorescein after Administration to Rat Skin by DMs

\begin{tabular}{|c|c|c|c|}
\hline DM & Dose $(\mathrm{mg})$ & $\begin{array}{l}\text { Cumulative } \\
\text { amount of fluores- } \\
\text { cein for } 6 \mathrm{~h}(\mathrm{mg})\end{array}$ & Permeability (\%) \\
\hline $\begin{array}{l}\text { FLNa DM, type } \\
\text { (1) }\end{array}$ & $0.18 \pm 0.03$ & $0.17 \pm 0.03$ & $96.4 \pm 14.4$ \\
\hline $\begin{array}{l}\text { FLNa DM, type } \\
\text { (2) }\end{array}$ & $0.64 \pm 0.07$ & $0.46 \pm 0.06$ & $71.9 \pm 9.5$ \\
\hline $\begin{array}{l}\text { FLNa DM, type } \\
\text { (3) }\end{array}$ & $10.95 \pm 1.07$ & $2.95 \pm 0.92$ & $26.9 \pm 8.4$ \\
\hline $\begin{array}{l}\text { FL DM, type } \\
\text { (1) }\end{array}$ & $0.20 \pm 0.01$ & $0.005 \pm 0.002$ & $2.7 \pm 0.8$ \\
\hline $\begin{array}{l}\text { FL DM, type } \\
\text { (2) }\end{array}$ & $0.68 \pm 0.03$ & $0.014 \pm 0.002$ & $2.0 \pm 0.3$ \\
\hline $\begin{array}{l}\text { FL DM, type } \\
\text { (3) }\end{array}$ & $12.47 \pm 1.01$ & $0.038 \pm 0.008$ & $0.3 \pm 0.1$ \\
\hline
\end{tabular}

Each value represents the mean \pm S.D. $(n=5)$. permeablity $(\%)=$ cumulative permeated amount of fluorescein for $6 \mathrm{~h}(\mathrm{mg}) /$ applied amount $(\mathrm{mg}) \times 100$.

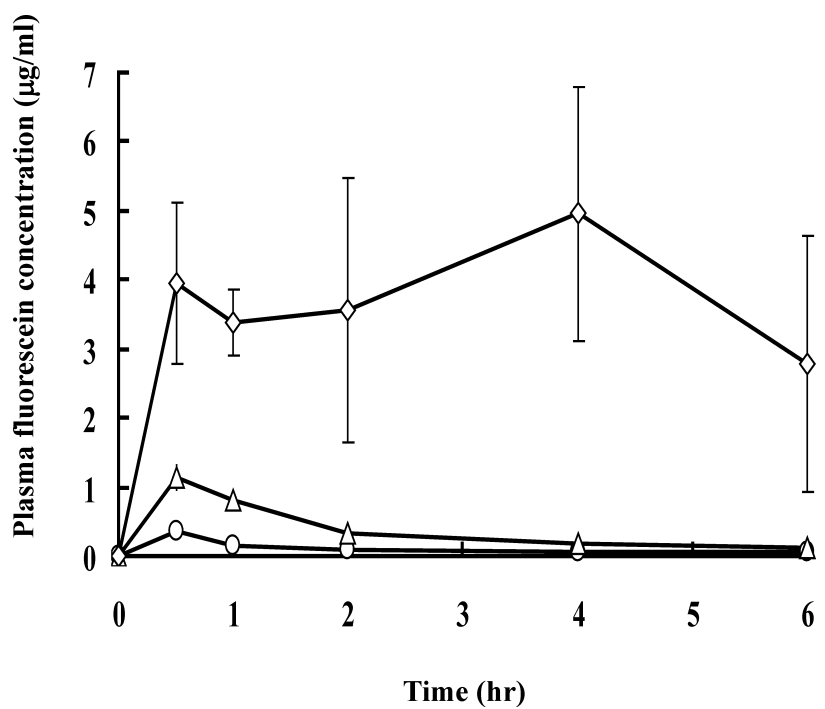

Fig. 5. Plasma Fluorescein Concentration-Time Curves after Percutaneous Administration of FLNa DM Array Chips to Rats

$\bigcirc$ : type (1) DMs, $\triangle$ : type (2) DMs, $\diamond$ : type (3) DMs. Each point shows the mean \pm S.D. of 4 experiments. 
skin from FL loaded DMs was increased, $0.17 \pm 0.03 \mathrm{mg}$ for type (1) DMs, $0.46 \pm 0.06 \mathrm{mg}$ for type (2) DMs and 2.95 $0.92 \mathrm{mg}$ for type (3) DMs, respectively, as the loaded amount of FLNa were increased. The cumulative permeated amounts of fluorescein through the rat skin from FL loaded DMs was $0.005 \pm 0.002 \mathrm{mg}$ for type (1) DMs, $0.014 \pm 0.002 \mathrm{mg}$ for type (2) DMs, and $0.038 \pm 0.008 \mathrm{mg}$ for type (3) DMs. Results show that the permeated amount of fluorescein from FLNa loaded DMs were 32-77 times higher than those of the FL loaded DMs as shown in Table 1. Therefore, FLNa loaded DM array chips were used for the following in vivo evaluation experiment using rats.

Systemic Delivery of Fluorescein in Rats DM array chips of three kinds, types (1)-(3), loaded with FLNa were administered to the hair-removed rat abdominal skin and plasma fluorescein levels were measured as presented in Fig. 5. Each DM preparation showed, respectively, the $C_{\max }$ of $0.36 \pm 0.02$, $1.14 \pm 0.17$ and $3.96 \pm 1.17 \mu \mathrm{g} / \mathrm{mL}$. $T_{\max }$ were $0.5 \mathrm{~h}, 0.5 \mathrm{~h}$ and 4h. Table 2 shows that $A U C_{0-6}$ were $0.72 \pm 0.08,2.16 \pm 0.98$ and $22.5 \pm 9.32 \mu \mathrm{g} \cdot \mathrm{h} / \mathrm{mL}$. To obtain the absorbed amount of fluorescein into the systemic circulation, an i.v. experiment was performed with another group of rats, $1.0 \mathrm{mg} / \mathrm{kg}$. The $A U C_{0-\infty}$ was $1.37 \pm 0.04 \mu \mathrm{g} \cdot \mathrm{h} / \mathrm{mL}$. By comparing the $A U C$ values obtained after percutaneous administration of DMs to that obtained after i.v. injection of fluorescein solution, the amount of fluorescein absorbed into the systemic circulation was estimated and the results are also shown in the table. Because the loaded amount of FLNa increased from type (1) to type (3) DMs, the absorbed amount of fluorescein was increased

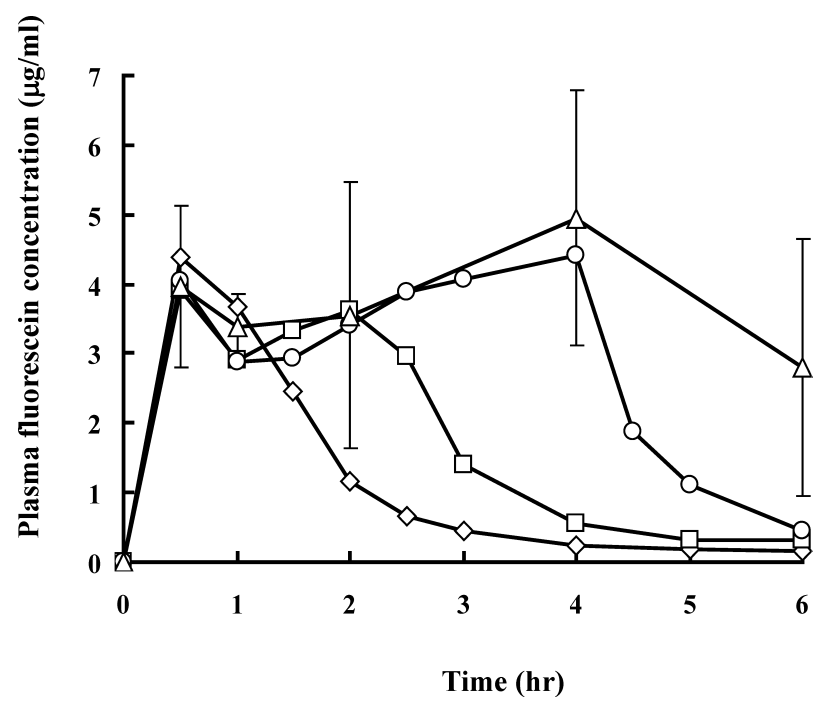

Fig. 6. Effect of Application Time, $(\diamond)$ for $1 \mathrm{~h},(\square)$ for $2 \mathrm{~h}$, $(\bigcirc)$ for $4 \mathrm{~h}$ and $(\triangle)$ for $6 \mathrm{~h}$, on Plasma Fluorescein Concentration-Time Curves after Percutaneous Administration of FLNa DM to Rats

Each point shows the mean \pm S.D. of 4 experiments. from $0.18 \pm 0.02 \mathrm{mg}$ and $0.53 \pm 0.19 \mathrm{mg}$, to $5.38 \pm 1.99 \mathrm{mg}$. The bioavailabilities of fluorescein from three DMs were 95.6 $11.4,81.3 \pm 8.2$ and $50.3 \pm 16.3 \%$, respectively. The type (3) DM had a large amount of loss of drugs ascribed to the drug layer in tablet. Although the abosorbed amount of FLNa was higher than type (2), lower bioavailability was obtained.

Effect of Application Time of DMs on Systemic Absorption of Fluorescein The type (3) DM array chips were administered to the rat abdominal skin. The chips were fixed with adhesive tape for four different times, 1, 2, 4, and $6 \mathrm{~h}$, and plasma pharmacokinetic examination was performed. Figure 6 presents the results: for decreased application times from 6 to $1 \mathrm{~h}$, the $A U C$ decreased, 22.5 $49.3,16.9 \pm 2.8,10.5 \pm$ 3.7, and $7.0 \pm 0.9 \mu \mathrm{g} \cdot \mathrm{h} / \mathrm{mL}$ (Table 3). The amounts fluorescein absorbed into the systemic circulation were estimated respectively as $5.34 \pm 1.99 \mathrm{mg}, 4.26 \pm 0.68,2.52 \pm 0.89$, and $1.70 \pm$ $0.22 \mathrm{mg}$.

Recovery Rate of Skin Damage To study the recovery of the skin damage induced by the administration of DMs, the

Table 3. Pharmacokinetic Parameters of Fluorescein after Percutaneous Administration of Type (3) FLNa MN to Rat Skin for Different Application Time

\begin{tabular}{cccc}
\hline \hline $\begin{array}{c}\text { Application period } \\
(\mathrm{h})\end{array}$ & $\begin{array}{c}A U C_{0-6} \\
(\mu \mathrm{g} \cdot \mathrm{h} / \mathrm{mL})\end{array}$ & $\begin{array}{c}\text { Amount absorbed } \\
\text { for 6h }(\mathrm{mg})\end{array}$ & BA (\%) \\
\hline 1 & $7.03 \pm 0.91$ & $1.70 \pm 0.22$ & $15.6 \pm 2.0$ \\
2 & $10.46 \pm 3.68$ & $2.52 \pm 0.89$ & $23.1 \pm 8.0$ \\
4 & $16.91 \pm 2.82$ & $4.26 \pm 0.68$ & $37.8 \pm 6.1$ \\
6 & $22.51 \pm 9.32$ & $5.34 \pm 1.99$ & $50.3 \pm 16.3$ \\
\hline
\end{tabular}

$A U C_{0-6}:$ Area under the serum fluorescein concentration $v s$. time curve from time zero to $6 \mathrm{~h}$. BA: Bioavailability. Each value represents the mean \pm S.D. $(n=4)$.

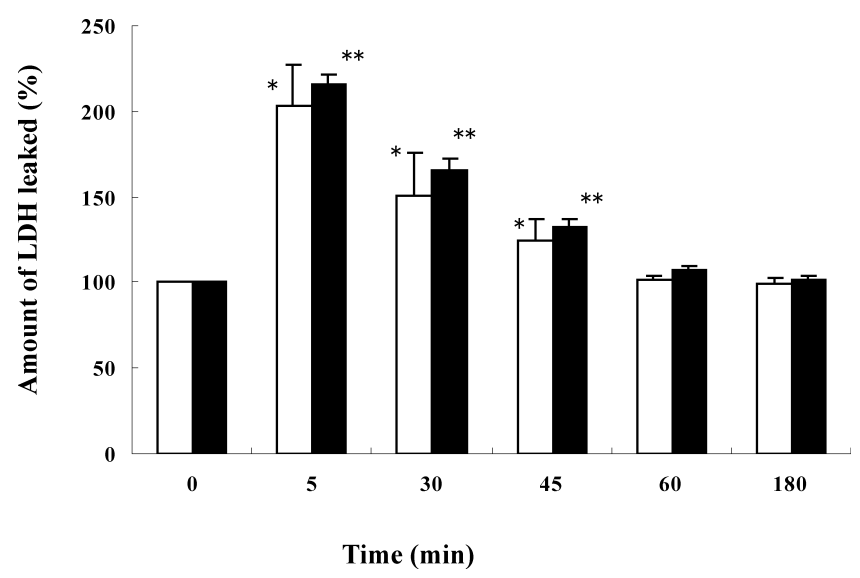

Fig. 7. Effect of Application Time, $(\square)$ for $3 \mathrm{~min}$ and ( $\square$ ) for $6 \mathrm{~h}$, on LDH Leakage from the Administered Skin after Percutaneous Administration of FLNa DM to Rats

Each point shows the mean \pm S.D. of 4 experiments. *: $p<0.05$ significantly different from control value. **: $p<0.05$ significantly different from $3 \mathrm{~min}$.

Table 2. Pharmacokinetic Parameters of Fluorescein after Percutaneous Administration of FLNa DMs to Rat Skin

\begin{tabular}{|c|c|c|c|c|c|c|}
\hline $\mathrm{DM}$ & Dose (mg) & $C_{\max }(\mu \mathrm{g} / \mathrm{mL})$ & $T_{\max }(\mathrm{h})$ & $A U C_{0-6}(\mu \mathrm{g} \cdot \mathrm{h} / \mathrm{mL})$ & $\begin{array}{l}\text { Amount absorbed for } \\
6 \mathrm{~h}(\mathrm{mg})\end{array}$ & BA (\%) \\
\hline Type (1) & $0.18 \pm 0.03$ & $0.36 \pm 0.02$ & 0.5 & $0.72 \pm 0.08$ & $0.18 \pm 0.02$ & $95.6 \pm 11.4$ \\
\hline Type (2) & $0.64 \pm 0.07$ & $1.14 \pm 0.17$ & 0.5 & $2.16 \pm 0.98$ & $0.53 \pm 0.19$ & $81.3 \pm 8.21$ \\
\hline Type (3) & $10.95 \pm 1.07$ & $3.96 \pm 1.17$ & 4.0 & $22.51 \pm 9.32$ & $5.38 \pm 1.99$ & $50.3 \pm 16.3$ \\
\hline
\end{tabular}

$C_{\mathrm{max}}$ : The maximum value of plasma fluorescein level. $T_{\max }$ : The time when plasma fluorescein level reaches to its maximum value. $A U C_{0-6}:$ Area under the plasma fluorescein concentration $v s$. time curve from time zero to $6 \mathrm{~h}$. BA: Bioavailability. Each value represents the mean \pm S.D. $(n=4)$. 

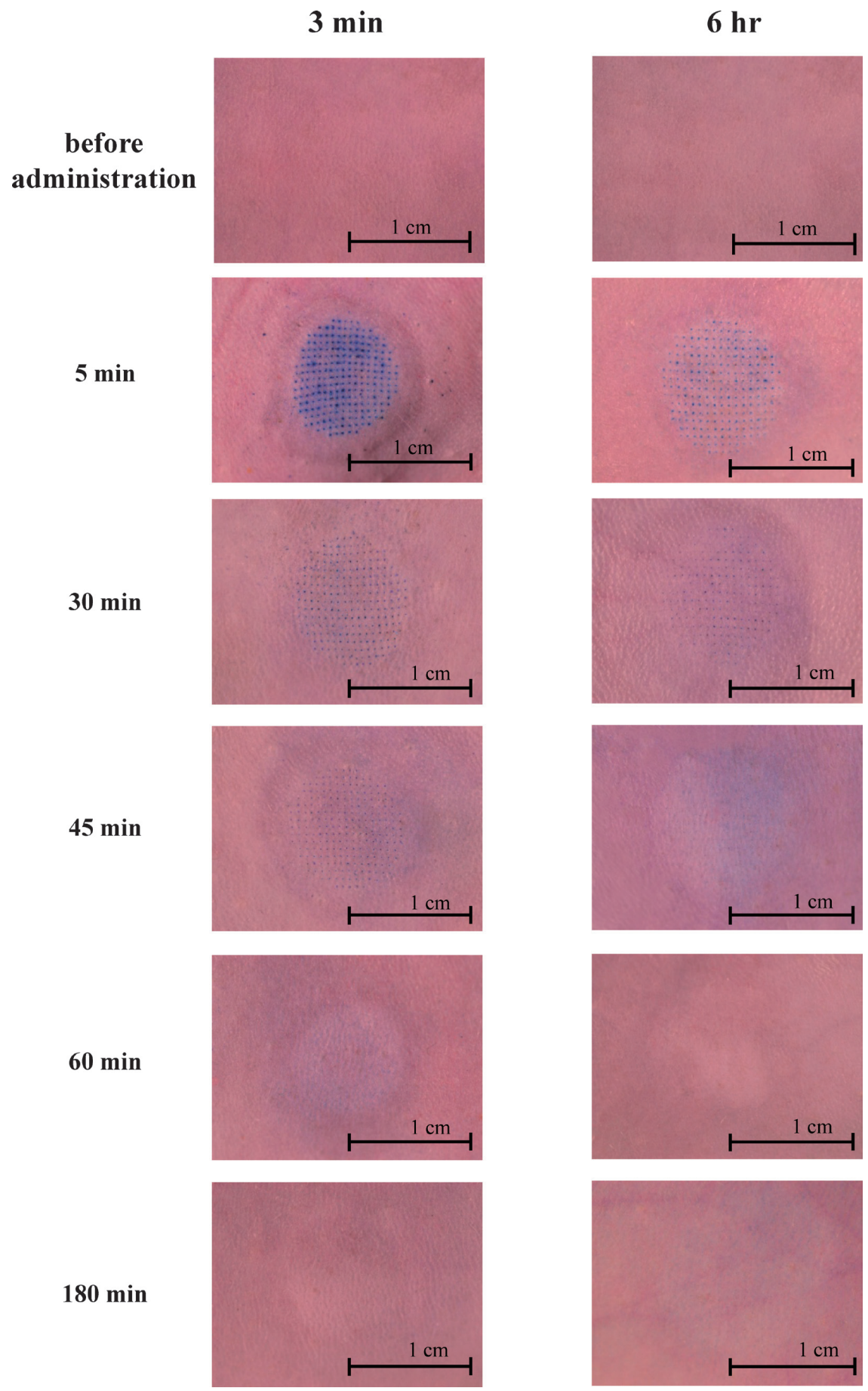

Fig. 8. Pathological Examination of the Skin Staining with 1.0\% Evans Blue Solution after the Percutaneous Administration of FLNa DM to Rats

leakage of LDH from the administered skin was measured with two groups of rats, one group received DMs for $3 \mathrm{~min}$ and the second group received them for $6 \mathrm{~h}$. The results are shown in Fig. 7. In both groups, the leaked amounts of LDH were significantly increased at $30 \mathrm{~min}$ after the administration of DMs. However, the LDH levels returned to the pre-dose level at $60 \mathrm{~min}$, because there were not significant differences against the pre-dose levels in both groups. Figure 8 shows the results on the pathological examination of the skin. In both groups, the staining disappeared at $60 \mathrm{~min}$ after the administration of DMs in both groups.

\section{Discussion}

Using DMs, the permeability of drugs through the skin was improved dramatically and high BAs of many biopharmaceuticals were obtained, $91.3-97.7 \%$ for insulin in mice $^{13)}$ and of $81.5-102.3 \%$ for LMWH in rats, ${ }^{14)} 87.5 \%$ for rhGH in rats ${ }^{15)}$ and of $82.1-99.4 \%$ for EPO in mice. ${ }^{16)}$ The relative BA of IFN was $79.9-117.8 \%$ in rats. ${ }^{17)}$ The relative physiological availability (PA) of insulin was $90-99 \%$ in dogs. ${ }^{18)}$ However, the clinical doses of those drugs are lower than $1.0 \mathrm{mg}$. To increase the application range of DM technology to drugs of which the clinical dose is higher than $1.0 \mathrm{mg}$, we must make a breakthrough in DMs. In this study, we prepared DM array chips of 
three kinds, (1) drug is localized at the acral portion of DMs, (2) drug is loaded in the whole DMs, and (3) drug is loaded both DMs and chip. By increasing the formulated space of drug in DM array chip, the permeated amount of drug through the skin was increased. Comparing with in vitro dissolution profiles, there was a lag time ascribed to the water condition differences. However, a difference existed on the permeation enhancing effect between the drugs, FLNa and FL. When free form of fluorescein, FL, was used, the permeation enhancing effect was not high, $0.3-2.67 \%$ for $6 \mathrm{~h}$. When its sodium salt, FLNa, was used, permeability was 32-77 times increased. Therefore, we might state that the microconduits formed by the insertion of DMs made the dissolved drug molecules to access the stratum corneum directly and to permeate the skin with high efficiency, as Chabri et al. pointed out. ${ }^{25)}$

The mainly applicable drugs of DMs as TDDS are biopharmaceuticals such as peptide proteins including insulin. Biopharmaceuticals are fundamentally water-soluble, i.e. hydrophilic, substances. ${ }^{26)}$ The skin has a strong barrier function. Therefore hydrophilic compounds are not absorbed through the skin. However, DMs physically break the barrier function of the skin. Therefore, biopharmaceuticals were absorbed into the skin, though they are hydrophilic macromolecules. In this study, biopharmaceuticals were not used because of their low clinical dose attributable to their high pharmacological activities. Instead, FLNa and FL were used as model drugs of both water-soluble and water-insoluble compounds at physiological pH. FLNa is used for diagnosis in ophthalmology. However, FL is the free form of fluorescein. It is not soluble at the neutral $\mathrm{pH}$ range. As inferred from their solubility at physiological $\mathrm{pH}$, the dissolution rate of fluorescein from $\mathrm{FL}$ DMs was far slower than FLNa DMs. In addition, the rate of permeability of fluorescein through the rat skin from FL DMs was approximately $1 / 10$ as compared to that obtained after application of FLNa DMs. Therefore, DMs of three kinds were evaluated in rat experiment with FLNa. Because the formulated amount of FLNa in the DM array chip increased from 0.18 to $10.95 \mathrm{mg}$, the absorbed amount of fluorescein into the systemic circulation increased from 0.18 to $5.38 \mathrm{mg}$. As FLNa was dissolved easily from the array chip and permeated the rat skin through the microconduit formed on the skin surface. Therefore, type (3) DM array chip was revealed to be inapplicable to increase the systemic delivery of water-insoluble hydrophobic drug. Instead, type (3) DM array chip is a useful system for the systemic delivery of water-soluble hydrophilic drug. The DM array chip used in this study had $225 \mathrm{DMs}$ and caused the systemically delivered amount of $5.34 \mathrm{mg}$ of fluorescein. A 15-mm-diameter chip was used. By increasing the chip diameter to $25 \mathrm{~mm}$, the number of DMs formed on the chip can be increased to 450 . Therefore, a few tens of milligrams of fluorescein which covers most clinical use drugs, can be delivered into the systemic circulation by this system. Because the duration of the opening of the microconduit in the skin is the key factor affecting drug permeation through the skin, we must check the side effect of skin irritation that occurs when DMs are applied to the skin for $6 \mathrm{~h}$. Both biochemical and pathological studies showed that the skin physiology recovered to the normal level within $60 \mathrm{~min}$ after the removal of the DM array chips. Prausnitz and colleagues studied the duration of microconduit opening by microneedles. Their results showed that $4 \mathrm{~h}$ was necessary for microconduits to close after the formation of microconduit by microneedles. ${ }^{27}$ We studied the effect of the DM application duration on the in vivo permeation of FLNa through rat skin by measuring the plasma fluorescein levels. Although the application time was decreased from 6 to $1 \mathrm{~h}$, the plasma fluorescein level vs. time curves showed the same pattern and the absorbed amount of drug decreased from 5.34 to $1.7 \mathrm{mg}$. From those results, we can infer that the microconduits formed by DMs were opened until the DM array chip was removed from the rat skin and fluorescein was absorbed through the skin as long as the chip was attached to the skin. Both histological and biochemical studies were conducted to investigate the recovery time of the microconduit formed on the skin. No difference was found in the recovery time of the rat skin between the application times of $5 \mathrm{~min}$ and $6 \mathrm{~h}$, judging from the skin images obtained by evans blue staining and LDH leakage. Therefore, we might state that long-term application of the DM array chip to the skin would not affect the clinical application of DMs. The dissolved drug molecules were able to permeate the skin as long as the chip was attached to the skin.

\section{Conclusion}

To increase the systemically delivered amount of drug, two fluorescein compounds, sodium fluorescein (FLNa), and free form (FL), were used as model water-soluble and water-insoluble compounds to prepare dissolving microneedles (DMs) of three types, in which (1) the drug is localized at the acral portion of DMs, (2) the drug is loaded in the whole DMs, or (3) the drug is loaded both DMs and chip. The in vitro permeability of fluorescein through the rat skin from FL loaded DMs was much lower than FLNa loaded DMs because of the slower dissolution rate of fluorescein from FL loaded DMs. The systemic delivery efficiency of three types of FLNa loaded DMs was evaluated in the in vivo rat absorption experiment. By applying DM array chip to the rat abdominal skin for $6 \mathrm{~h}$, systemically absorbed amount of fluorescein increased from 0.18 to 0.53 and $5.38 \mathrm{mg}$ from type (1)-(3) DM array chips. The systemically delivered amount of drug was dependent on the application time of chips to the skin. The physiological barrier function of the skin recovered within $30 \mathrm{~min}$ after removal of the chips. The type (3) DM array chip system is useful for the delivery of more than $1.0 \mathrm{mg}$ of the drug to the systemic circulation.

Acknowledgements This study was supported by a Ministry of Education, Culture, Sports, Science and Technology (MEXT) of Japan-Supported Program for the Strategic Research Foundation at Private Universities, 2008-2013. This study was also supported by a Grant-in-Aid for Scientific Research provided by MEXT, 2010-2013.

\section{References}

1) Guy R. H., "Transdermal drug delivery, in Handbook of Experimental Pharmacology 197: Drug Delivery," ed. by Schafer-Korting M., Springer, Berlin, 2004, pp. 399-410.

2) Barry B., Williams A., Adv. Drug Deliv. Rev., 56, 603-618 (2004).

3) Cevc G., Adv. Drug Deliv. Rev., 56, 675-711 (2004).

4) Preat V., Vanbever R., Adv. Drug Deliv. Rev., 56, 659-674 (2004).

5) Doukas A. G., Kollias N., Adv. Drug Deliv. Rev., 56, 559-579 (2004).

6) Mitragotri S., Kost J., Adv. Drug Deliv. Rev., 56, 589-601 (2004). 
7) Wermeling D. P., Banks S. L., Hudson D. A., Gill H. S., Gupta J., Prausnitz M. R., Stinchcomb A. L., Proc. Natl. Acad. Sci. U.S.A., 105, 2058-2063 (2008).

8) Cormier M., Johnson B., Ameri M., Nyam K., Libiran L., Zhang D. D., Daddona P., J. Controlled Release, 97, 503-511 (2004).

9) Prausnitz M. R., Adv. Drug Deliv. Rev., 56, 581-587 (2004).

10) Levin G., Gershonowitz A., Sacks H., Stern M., Sherman A., Rudaev S., Zivin I., Phillip M., Pharm. Res., 22, 550-555 (2005).

11) Takada K. Drug., Drug. Discov. Ther., 2, 140-155 (2008).

12) Ito Y., Murakami A., Maeda T., Sugioka N., Takada K., Int. J. Pharm., 349, 124-129 (2008).

13) Ito Y., Hagiwara E., Saeki A., Sugioka N., Takada K., Eur. J. Pharm. Sci., 29, 82-88 (2006).

14) Ito Y., Murakami A., Maeda T., Sugioka N., Takada K., Int. J. Pharm., 349, 124-129 (2008).

15) Ito Y., Ohashi Y., Shiroyama K., Sugioka N., Takada K., Biol. Pharm. Bull., 31, 1631-1633 (2008).

16) Ito Y., Yoshimitsu J., Shiroyama K., Sugioka N., Takada K., J. Drug Target., 14, 255-261 (2006).

17) Ito Y., Saeki A., Shiroyama K., Sugioka N., Takada K., J. Drug Target., 16, 243-249 (2008).
18) Ito Y., Ohashi Y., Saeki A., Sugioka N., Takada K., Chem. Pharm. Bull., 56, 243-246 (2008).

19) Ito Y., Hasegawa R., Fukushima K., Sugioka N., Takada K., Biol. Pharm. Bull., 33, 683-690 (2010).

20) Fukushima K., Ise A., Morita H., Hasegawa R., Ito Y. Sugioka N., Takada K., Pharm. Res., 28, 7-21 (2011).

21) Ito Y., Murano H., Hamasaki N., Fukushima K., Takada K., Int. J. Pharm., 407, 126-131 (2011).

22) Ito Y., Kashiwara S., Fukushima K., Takada K., Drug Dev. Ind. Pharm., 37, 1387-1393 (2011).

23) Song Y., Xiao C., Mendelsohn R., Zheng T., Strekowski L., Michniak B., Pharm. Res., 22, 1918-1925 (2005).

24) Ito Y., Nakahigashi T., Yoshimoto N., Ueda Y., Hamasaki N., Takada K., Diabetes Technol. Ther., 14, 891-899 (2012).

25) Chabri F., Bouris K., Jones T., Barrow D., Hann A., Allender C., Brain K., Birchall J., Br. J. Dermatol., 150, 869-877 (2004).

26) Walsh G., "Biopharmaceuticals," John Wiley \& Sons, West Sussex, 2003.

27) Gupta J., Gill H. S., Andrews S. N., Prausnitz M. R., J. Controlled Release, 154, 148-155 (2011). 